\title{
Pore-scale imaging of geological carbon dioxide storage under in situ conditions
}

\author{
Matthew Andrew, ${ }^{1}$ Branko Bijeljic, ${ }^{1}$ and Martin J. Blunt ${ }^{1}$ \\ Received 30 April 2013; revised 13 July 2013; accepted 17 July 2013; published 7 August 2013.
}

[1] While geological carbon dioxide $\left(\mathrm{CO}_{2}\right)$ storage could contribute to reducing global emissions, it must be designed such that the $\mathrm{CO}_{2}$ cannot escape from the porous rock into which it is injected. An important mechanism to immobilize the $\mathrm{CO}_{2}$, preventing escape, is capillary trapping, where $\mathrm{CO}_{2}$ is stranded as disconnected pore-scale droplets (ganglia) in the rock, surrounded by water. We used X-Ray microtomography to image, at a resolution of $6.4 \mu \mathrm{m}$, the pore-scale arrangement and distribution of trapped $\mathrm{CO}_{2}$ clusters in a limestone. We applied high pressures and temperatures typical of a storage formation, while maintaining chemical equilibrium between the $\mathrm{CO}_{2}$, brine, and rock. Substantial amounts of $\mathrm{CO}_{2}$ were trapped, with an average saturation of 0.18 . The cluster sizes obeyed a power law distribution, with an exponent of approximately -2.1 , consistent with predictions from percolation theory. This work confirms that residual trapping could aid storage security in carbonate aquifers. Citation: Andrew, M., B. Bijeljic, and M. J. Blunt (2013), Porescale imaging of geological carbon dioxide storage under in situ conditions, Geophys. Res. Lett., 40, 3915-3918, doi:10.1002/grl.50771.

\section{Introduction}

[2] The original work on 3-D X-ray microtomography ( $\mu \mathrm{CT})$ by Flannery et al. [1987] states in conclusion: "we believe that it will be possible to study contained systems under conditions of temperature, pressure, and environment representative of process conditions." Since this seminal work, there has been an explosion in the use of $\mu \mathrm{CT}$ with the rapid development of bench-top scanners and huge interest in the study of porous rocks - as in the original paper-with application to hydrocarbon production and carbon dioxide storage. $\mu \mathrm{CT}$ is now the foremost method for the noninvasive imaging of rock cores at ambient conditions, applied to modeling and experimental interpretation [Berg et al., 2013; Blunt et al., 2013; Dann et al., 2011; Feali et al., 2012; Wildenschild and Sheppard, 2013]; however, imaging under conditions representative of flow and transport deep underground, including effects due to chemical equilibrium, has remained a challenge [Silin et al.,

Additional supporting information may be found in the online version of this article.

${ }^{1}$ Qatar Carbonates and Carbon Storage Research Centre, Department of Earth Science and Engineering, Imperial College London, London, UK.

Corresponding author: M. Andrew, Qatar Carbonates and Carbon Storage Research Centre, Department of Earth Science and Engineering, South Kensington Campus, Imperial College London, London SW7 2AZ, UK. (m.andrew11@imperial.ac.uk)

(C)2013. American Geophysical Union. All Rights Reserved. 0094-8276/13/10.1002/grl.50771
2011]. In this paper, we present the first in situ images of multiple phases in the pore space at elevated temperatures and pressures, representative of an aquifer at around $1 \mathrm{~km}$ depth, while maintaining mutual chemical equilibrium between the fluid phases and the rock.

[3] The application of this study is for geological carbon dioxide $\left(\mathrm{CO}_{2}\right)$ storage, where the concern is to design injection such that the $\mathrm{CO}_{2}$ remains underground for hundreds to thousands of years. Sedimentary basins that are potentially suitable carbon storage sites include deep carbonate aquifers [Bachu, 2003]. An important mechanism that limits the spread and potential escape of $\mathrm{CO}_{2}$ is capillary trapping, where $\mathrm{CO}_{2}$, displaced by aquifer brine, is stranded as pore-scale droplets (ganglia) [Juanes et al., 2006]. Under favorable conditions, this process can, in theory, render the vast majority of the $\mathrm{CO}_{2}$ immobile [Ennis-King and Paterson, 2002; Golding et al., 2011; Qi et al., 2009]. The average amount of trapping can be measured in core flood ( $\mathrm{cm}$ scale) experiments [Akbarabadi and Piri, 2013; Bennion and Bachu, 2010; El-Maghraby, 2013; El-Maghraby and Blunt, 2013; Okabe and Tsuchiya, 2008; Pentland et al., 2011] and has been imaged at the pore scale in a sandstone [Iglauer et al., 2011]. However, in typical deep storage sites, the $\mathrm{CO}_{2}$ will be in a supercritical (sc) phase in mutual chemical equilibrium with the host brine and the rock: dissolved $\mathrm{CO}_{2}$ forms an acid that can react with many rock minerals, including carbonates. We demonstrate that, locally, substantial quantities of $\mathrm{scCO} 2$ can be trapped in the pore space of the limestone studied, at representative in situ conditions where the scCO2, brine, and rock are in mutual chemical equilibrium. We demonstrate that the trapped clusters have an approximately power law distribution of size, consistent with the predictions of percolation theory assuming that $\mathrm{CO}_{2}$ is the nonwetting phase [Blunt and Scher, 1995; Dias and Wilkinson, 1986]. It is also consistent with measurements of trapping on analogue systems at ambient conditions [Iglauer et al., 2012; Iglauer et al., 2010].

\section{Materials and Methods}

[4] We imaged $\mathrm{scCO}_{2}$ and brine phases in a homogenous limestone, Ketton Oolite, from the Upper Lincolnshire Limestone Member (deposited 169-176 million years ago) using an X-ray transparent flow apparatus and the Versa XRM-500 X-Ray Microscope (www.xradia.com). Four separate experiments were conducted. For details on the experimental apparatus and procedure, please refer to the supporting information. Ketton limestone has a bimodal pore structure, with a simple, cemented bead-pack like macropores and a micritic intragranular microporous structure.

[5] The raw reconstructed $\mu \mathrm{CT}$ scans were filtered using a nonlocal means edge preserving filter [Buades et al., 2005; 

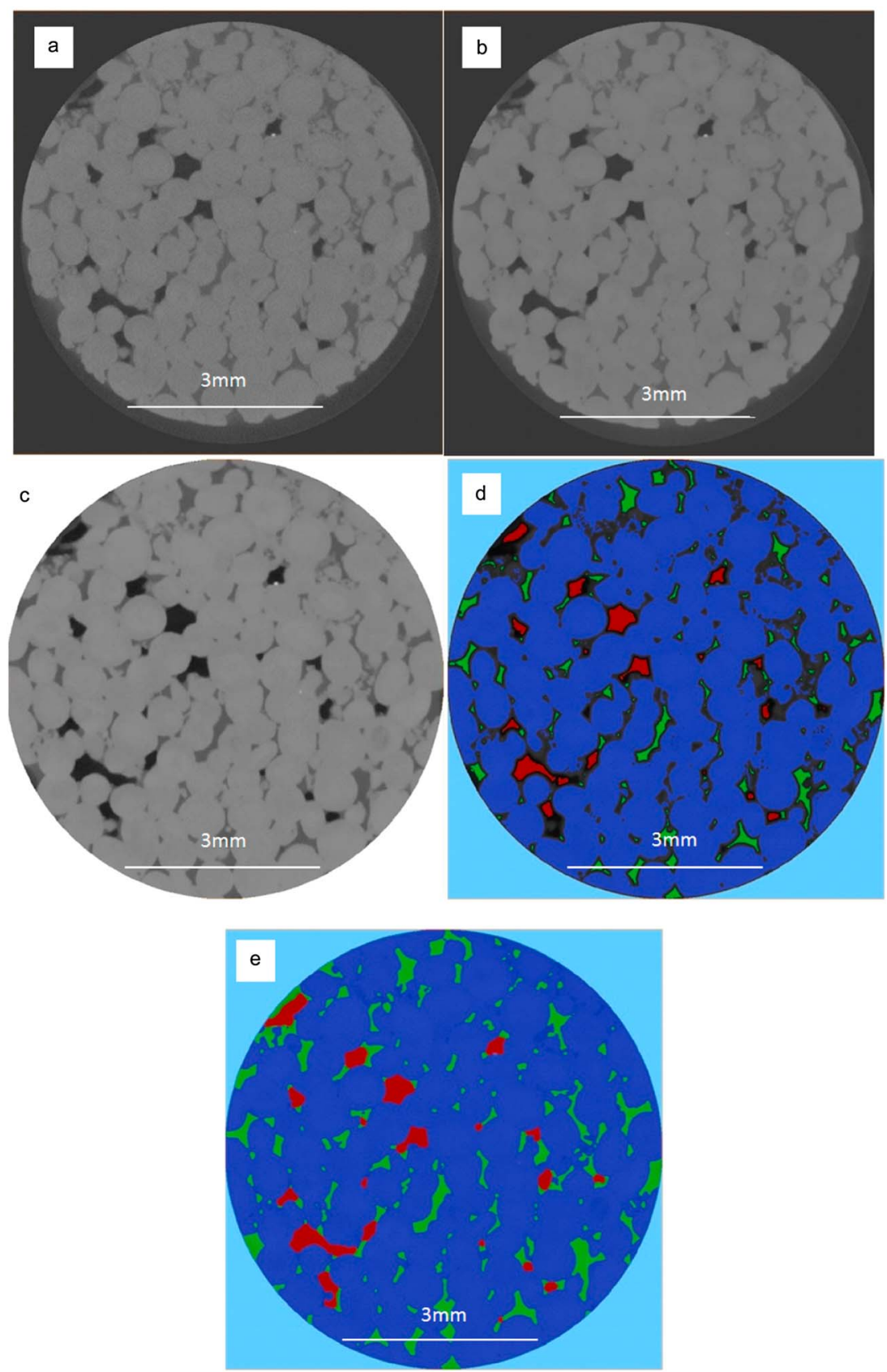

Figure 1. Image processing, consisting of four steps; $(a-b)$ filtering, $(b-c)$ cropping, $(c-d)$ watershed seed generation, and the (d-e) application of the watershed algorithm. The images show $\mathrm{scCO}_{2}$ (the darkest phase, red), brine (the intermediate phase, green), and the rock grains (the lightest phase, blue). The rock grains are around $700 \mu \mathrm{m}$ across.

2008] (Figures 1a-1b) to reduce noise. The images were then segmented using a seeded watershed algorithm (Figures 1c$1 \mathrm{e})$, based on the 3-D gradient magnitude and grey-scale value of each voxel. This eliminates much of the voxel misidentification present in simple grey-scale segmentation, as well as the arbitrary nature of the thresholds taken. It also eliminates partial volume artefacts. All image processing was conducted within the Avizo Fire 7.0 program (Visualization Sciences Group, www.vsg3d.com).

\section{Results}

[6] The separated phases were analyzed to compute the average saturation and the 3-D distribution of trapped
$\mathrm{CO}_{2}$, which resides in the pore space as isolated ganglia, surrounded by brine - see Figure 2 .

[7] The apparent porosity evaluated from the $\mu \mathrm{CT}$ image $(\varphi=0.1373)$ was lower than that measured using helium porosimetry (0.2337); $\mu \mathrm{CT}$ will only image pores larger than the image resolution and does not capture the microporosity within the oolite grains. From the image analysis, we compute the total number of voxels containing trapped $\mathrm{CO}_{2}$ : knowing the total volume of the image, we can compute the fraction of the rock volume containing $\mathrm{CO}_{2}$-this is the capillary trapping capacity. The saturation values were found by dividing by the total porosity of the sample. The oolite grains - and hence the microporosity-remained brinesaturated throughout the experiment; $\mathrm{CO}_{2}$ was prevented 


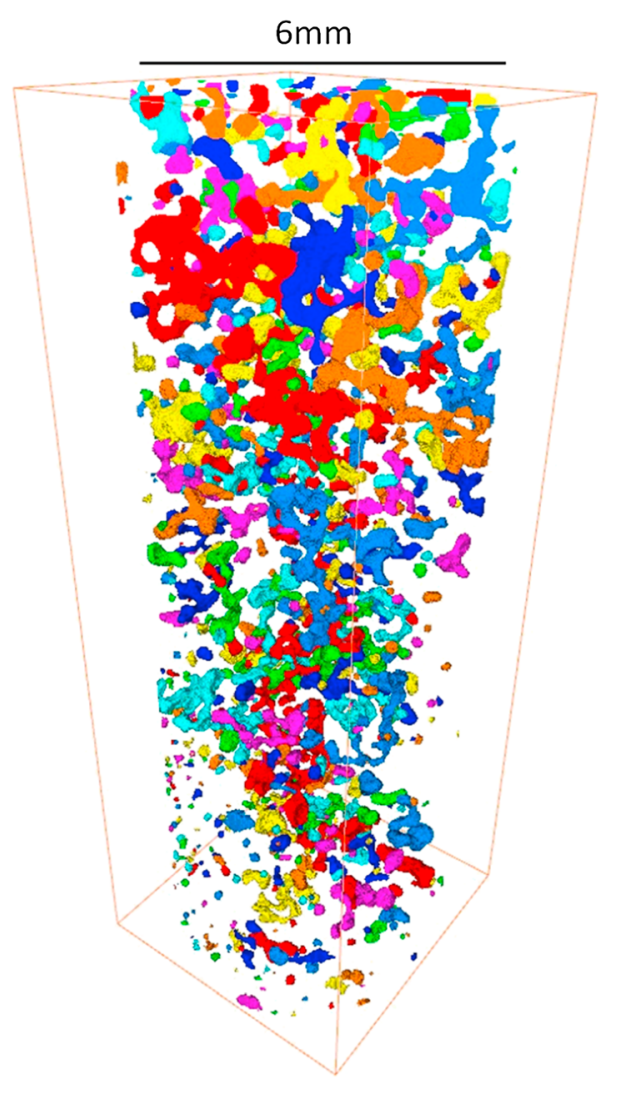

Figure 2. The 3-D rendering of experiment 2 after brine injection. Each unique $\mathrm{CO}_{2}$ ganglion is displayed as a different color. Each ganglion is isolated, and so is trapped.

from entering these regions because of its high capillary entry pressure ( $>0.1 \mathrm{MPa}$, from mercury injection analysis) compared to the maximum capillary pressures generated during the experiment $(<0.01 \mathrm{MPa})$.

[8] The volume-averaged residual (trapped) $\mathrm{CO}_{2}$ saturation was $0.19 \pm 0.03$, representing a capillary trapping capacity of $0.045 \pm 0.006$ : some $30-40 \%$ of the macroporosity contained trapped $\mathrm{CO}_{2}$. Similar experiments were performed on a larger cm-sized Ketton core [El-Maghraby, 2013]: averaged over five experiments, the residual saturation was $0.137 \pm 0.012$, with a capillary trapping capacity of $0.032 \pm 0.003$. While the values are comparable, we see more trapping in the microflow cell. This could be due to finite size effects and the confined flow domain that prevents the displacement of some $\mathrm{CO}_{2}$ that might be mobile in a larger system.

\section{Ganglia Size Analysis}

[9] At the pore scale, brine injection is an imbibition process, where a wetting fluid displaces a nonwetting phase. The wetting phase fills regions of the pore space in order of size, occupying the smallest pores first [Roof, 1970]. The nonwetting phase $\left(\mathrm{scCO}_{2}\right)$ is trapped as a result of a percolation-like process [Dias and Wilkinson, 1986]. If this is the case, then we expect to see trapped clusters of all size with a power law distribution: the number $N$ of clusters of volume $s$ (measured in voxels) should scale as $N(s) \sim s^{-\tau}$, with an exponent $\tau=2.189$ [Lorenz and Ziff, 1998]. There is a lower cutoff, since we do not expect to see clusters smaller than a typical pore size, and an upper cutoff, since the trapped clusters cannot be larger than the system size. To quantify this, the distribution of pore radii was computed from a topological analysis of the pore space that identifies the largest voids [Dong and Blunt, 2009]. A typical pore radius is around $40 \mu \mathrm{m}$ - consistent with the images shown in Figure 1giving a lower cutoff for $N(s)$ of around 2000 voxels.

[10] Figure 3 shows $C(s)=\int_{s}^{\infty} N(s) \mathrm{d} s / \int_{0}^{\infty} N(s) \mathrm{d} s \sim s^{1-\tau}$ for the different experiments. The ganglion size distribution gives information about the dynamic processes which created the residual ganglia and also the scale range relevant to the length scale of ganglia contributing to the residual saturation. The best fit to a power law for $\mathrm{s}>2000$ is shown obtained using the maximum likelihood estimator which helps to remove biases due to a small number of very large ganglia [Clauset et al., 2009]: the exponents obtained are shown in Table 2. In these experiments, the single largest ganglion did not contribute substantially to the overall trapped volume-indeed, since $\tau>2$, the residual saturation was dominated by the smallest clusters (those extending over just one or a few pores). This result is consistent with other studies on sandstones [Iglauer et al., 2011], but not simplified systems such as sintered glass splinters [Georgiadis et al., 2011], where, possibly, the displacement is not a percolation-like process. Our average exponent is $2.10 \pm 0.14$, which is close to the theoretical value from percolation theory (2.189) and the results of pore-scale modeling for a

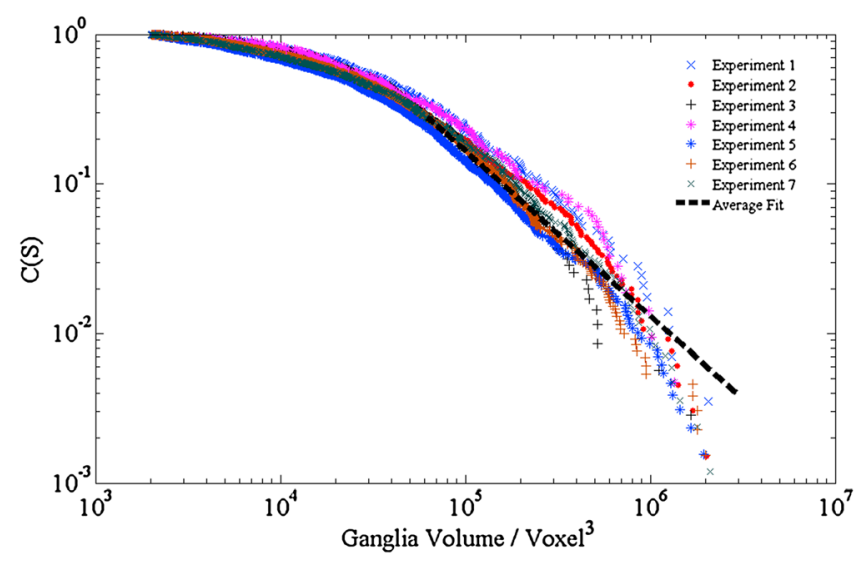

Figure 3. Cumulative ganglia size probability distributions for all experiments. 
strongly water-wet system (2.05) [Blunt and Scher, 1995]. This indicates that in the displacement process, $\mathrm{CO}_{2}$ behaves as a nonwetting phase, while the brine is the wetting phase. This confirms the conclusions of larger core floods on carbonates [El-Maghraby, 2013; El-Maghraby and Blunt, 2013], while contradicting some contact angle measurements of $\mathrm{scCO}_{2}$-brine-mineral systems that have suggested that under supercritical conditions, $\mathrm{scCO}_{2}$ may no longer be nonwetting [Espinoza and Santamarina, 2010; Jung and Wan, 2008; Kim et al., 2012; Yang et al., 2008], implying little opportunity for capillary trapping and-potentiallyreduced storage security [Chaulbaud et al., 2010].

\section{Conclusions}

[11] We have used a high-pressure high-temperature apparatus to image trapped supercritical $\mathrm{CO}_{2}$ in a carbonate system with a voxel size of $6.4 \mu \mathrm{m}$. The experiments employed a carbon fiber core holder to maintain pressure, while remaining largely X-ray transparent. The fluids and rock were preequilibrated to preserve conditions of chemical equilibrium throughout the experiments. The trapped clusters ranged in volume over 3 orders of magnitude, with an approximately power law distribution consistent with percolation theory, leading to a large surface area for dissolution and reaction. We suggest that the $\mathrm{CO}_{2}$ acts as the nonwetting phase, with a substantial fraction of the $\mathrm{CO}_{2}$ contained as isolated ganglia suggesting, locally, good storage security.

[12] This technique for high-resolution in situ imaging is easily applicable to other systems, and further work could extend this apparatus to study a wide variety of multiphase flow problems in porous media at conditions of temperature and pressure and geochemical equilibrium typical of storage aquifers, oil fields, or other deep geological systems.

[13] Acknowledgments. We gratefully acknowledge funding from the Qatar Carbonates and Carbon Storage Research Centre (QCCSRC), provided jointly by Qatar Petroleum, Shell, and Qatar Science \& Technology Park. We also thank the Imperial College Consortium on Pore-Scale Modeling for their support.

[14] The Editor thanks an anonymous reviewer for assisting in the evaluation of this paper.

\section{References}

Akbarabadi, M., and M. Piri (2013), Relative permeability hysteresis and capillary trapping characteristics of supercritical $\mathrm{CO}_{2}$ /brine systems: An experimental study at reservoir conditions, Adv. Water Res., 52, 190-206.

Bachu, S. (2003), Screening and ranking of sedimentary basins for sequestration of $\mathrm{CO}_{2}$ in geological media in response to climate change, Environ. Geol., 44(3), 277-289.

Bennion, D. B., and S. Bachu (2010), Drainage and imbibition $\mathrm{CO}_{2}$ /brine relative permeability curves at reservoir conditions for carbonate formations, SPE 134028, Proceedings of the SPE Annual Technical Conference and Exhibition.

Berg, S., et al. (2013), Real-time 3D imaging of Haines jumps in porous media flow, PNAS, 110(10), 3755-3759.

Blunt, M. J., and H. Scher (1995), Pore-level modeling of wetting, Physical Review E, 52(6), 6387-6403.

Blunt, M. J., B. Bijeljic, H. Dong, O. Gharbi, S. Iglauer, P. Mostaghimi, A. Paluszny, and C. Pentland (2013), Pore-scale imaging and modelling, Adv. Water Res., 51, 197-216.

Buades, A., B. Coll, and J.-M. Morel (2005), A non-local algorithm for image denoising, Computer Vision and Pattern Recognition.

Buades, A., B. Coll, and J.-M. Morel (2008), Nonlocal image and movie denoising, Int. J. Comput. Vision, 76(2), 123-139.
Chaulbaud, C., M. Robin, J. M. Lombard, J. Bertin, and P. Egermann (2010), Brine/ $\mathrm{CO}_{2}$ interfacial properties and effects on $\mathrm{CO}_{2}$ storage in deep saline aquifers, Oil and Gas Science and Technology - Rev. IFP, 65, 541-555.

Clauset, A., C. R. Shalizi, and M. E. J. Newman (2009), Power-law distributions in empirical data, SIAM Rev., 51(4), 661-703.

Dann, R., M. Turner, M. Close, and M. A. Knackstedt (2011), Multi-scale characterisation of coastal sand aquifer media for contaminant transport using X-ray computed tomography, Environmental Earth Sciences, 63(5), 1125-1137.

Dias, M. M., and D. Wilkinson (1986), Percolation with trapping, J. Phys. A: Math. Gen., 19, 3131-3146.

Dong, H., and M. J. Blunt (2009), Pore-network extraction from microcomputerized-tomography images, Physical Review E, 80.

El-Maghraby, R. (2013), Measurements of $\mathrm{CO}_{2}$ Trapping in Carbonate and Sandstone Rocks, Imperial College, London.

El-Maghraby, R. M., and M. J. Blunt (2013), Residual $\mathrm{CO}_{2}$ trapping in Indiana limestone, Environ. Sci. Technol., 47(1), 227-233.

Ennis-King, J., and L. Paterson (2002), Engineering aspects of geological sequestration of carbon dioxide, SPE 77809, Proceedings of the Asia Pacific Oil and Gas Conference and Exhibition.

Espinoza, D. N., and J. C. Santamarina (2010), Water- $\mathrm{CO}_{2}-$ mineral systems: Interfacial tension, contact angle, and diffusion-Implications to $\mathrm{CO}_{2}$ geological storage, Water Resour. Res., 46, W07537, doi:10.1029/ 2009WR008634.

Feali, M., W. V. Pinczewski, Y. Cinar, C. H. Ams, J. Y. Ams, M. Turner, T. Senden, N. Francois, and M. A. Knackstedt (2012), Qualitative and quantitative analyses of the three-phase distribution of oil, Water, and Gas in Bentheimer Sandstone by Use of Micro-CT Imaging SPE Reservoir Evaluation \& Engineering, 15(6), 706-711.

Flannery, B. P., H. W. Deckman, W. G. Roberge, and K. L. D'Amico (1987), Three-dimensional X-ray microtomography, Science, 237(4821), 1439-1444.

Georgiadis, A., S. Berg, G. Maitland, and H. Ott (2011), Pore-scale microCT imaging: Cluster size distribution during drainage and imbibition, SCA2011-59, Proceedings of the International Symposium of the Society of Core Analysts.

Golding, M. J., J. A. Neufeld, M. Hesse, and H. E. Huppert (2011), Twophase gravity currents in porous media, J. Fluid Mech., 678, 248-270.

Iglauer, S., S. Favretto, G. Spinelli, G. Schena, and M. J. Blunt (2010), X-ray tomography measurements of power-law cluster size distributions for the nonwetting phase in sandstones, Physical Review E, 82.

Iglauer, S., A. Paluszny, C. H. Pentland, and M. Blunt (2011), Residual $\mathrm{CO}_{2}$ imaged with X-ray micro-tomography, Geophys. Res. Lett., 38, L21403, doi:10.1029/2011GL049680.

Iglauer, S., M. Fernø, P. Shearing, and M. Blunt (2012), Comparison of residual oil cluster size distribution, morphology and saturation in oilwet and water-wet sandstone, J. Colloid Interface Sci., 375(1), 187-192.

Juanes, R., E. J. Spiteri, F. M. Orr Jr., and M. J. Blunt (2006), Impact of relative permeability hysteresis on geological $\mathrm{CO}_{2}$ storage, Water Resour. Res., 42, W12418, doi:10.1029/2005WR004806.

Jung, J., and J. Wan (2008), Supercritical $\mathrm{CO}_{2}$ and ionic strength effects on wettability of silica surfaces: Equilibrium contact angle measurements, Energy Fuels, 26, 6053-6059.

Kim, Y., J. Wan, T. J. Kneafsey, and T. K. Tokunaga (2012), Dewetting of silica surfaces upon reactions with supercritical $\mathrm{CO}_{2}$ and brine: Pore-scale studies in micromodels, Environ. Sci. Technol., 46, 4228-4235.

Lorenz, C. D., and R. M. Ziff (1998), Precise determination of the bond percolation thresholds and finite-size scaling corrections for the sc, fcc and bcc lattices, Physical Review E, 57(1), 230-236.

Okabe, H., and Y. Tsuchiya (2008), Experimental investigation of residual $\mathrm{CO}_{2}$ saturation distribution in carbonate rock, SCA2008-3, Proceedings of the International Symposium of the Society of Core Analysts.

Pentland, C. H., R. El-Maghraby, S. Iglauer, and M. J. Blunt (2011), Measurements of the capillary trapping of super-critical carbon dioxide in Berea sandstone, Geophys. Res. Lett., 38, L06401, doi:10.1029/ 2011 GL046683.

Qi, R., T. C. LaForce, and M. J. Blunt (2009), Design of carbon dioxide storage in aquifers, Int. J. Greenhouse Gas Control, 3, 195-205.

Roof, J. G. (1970), Snap-off of oil droplets in water-wet pores, SPE Journal, 10(1), 85-90.

Silin, D., L. Tomutsa, S. M. Benson, and T. W. Patzek (2011), Microtomography and pore-scale modeling of two-phase fluid distribution, Transp. Porous Media, 86, 495-515.

Wildenschild, D., and A. P. Sheppard (2013), X-ray imaging and analysis techniques for quantifying pore-scale structure and processes in subsurface porous medium systems, Adv. Water Res., 51, 217-246.

Yang, D., Y. Gu, and P. Tontiwachwuthikul (2008), Wettability determination of the reservoir brine-reservoir rock system with dissolution of $\mathrm{CO}_{2}$ at high pressures and elevated temperatures, Energy Fuels, 22, 504-509. 